\title{
Fabry-Perot filters used for optical preprocessing
}

\author{
G. Indebetouw and S. K. Case
}

\begin{abstract}
A Fabry-Perot etalon placed in the object plane of a coherent optical processing system can be used as a variable bandpass preprocessing filter. Use of this filter leads to improved correlation signals in a matched spatial filter system. Experimental results are shown and compared with results obtained by two other preprocessing methods.
\end{abstract}

\section{Introduction}

In coherent optical pattern recognition systems it would often be useful to eliminate most or all of the undiffracted light (dc) from the optical system. In matched spatial filtering, for example, object identification is invariably performed using moderate or high spatial frequency information. The intense dc or low spatial frequency signal, in addition to not being useful, can often be detrimental since it can lead to an appreciable amount of undesired light being diffracted or scattered into the correlation plane.

Toward the end of improving the signal-to-noise ratio and the selectivity of matched spatial filter optical systems, we have investigated three different methods of low spatial frequency suppression. The newest method, consisting of inserting a Fabry-Perot etalon in the object plane is shown to give the best results.

\section{Optical Systems}

Three optical systems were constructed for these experiments, each employing different principal methods of low spatial frequency suppression. For each system, the same input object was used to construct a matched spatial filter. An array of input test objects was then used to produce an array of autocorrelation and cross-correlation signals whose relative intensities were measured.

G. Indebetouw is with Virginia Polytechnic Institute \& State University, Physics Department, Blacksburg, Virginia 24061; S. K. Case is with University of Minnesota, Department of Electrical Engineering, Minneapolis, Minnesota 55455.

Received 24 January 1981.

0003-6935/81/091715-04\$00.50/0.

(C) 1981 Optical Society of America.

\section{A. System A}

The first system, shown in Fig. 1, is a conventional optical correlation system. In recording the matched spatial filter (MSF) for this system, the film is simply overexposed for low spatial frequencies. The filter attenuation is thus a monotonic function of the magnitude of the object power spectrum. This technique, of course, is not new ${ }^{1,2}$ but is shown for comparative purposes. The technique is simple to perform (in fact difficult to avoid) but not easy to control. It also suffers the disadvantage of having the low frequency suppression being sensitive to filter position.

The object used to construct the matched spatial filter (MSF) for the system was a 1-mm diam transparent circle [the middle size circle in Fig. 2(B)]. The object spectrum to reference beam intensity ratio and the film exposure time were chosen to maximize the filter diffraction efficiency for a spatial frequency in the object spectrum near 5 lines $/ \mathrm{mm}$.

The impulse response of the filter is shown in Fig. 2(A). Low frequency suppression is quite evident. An array of objects [Fig. 2(B)] was placed in the object plane to obtain the correlation signals of Fig. 2(C). In Fig. 2(D) an intensity measurement through the center row of output signals of Fig. 2(C) shows two relatively strong cross-correlation signals in addition to the autocorrelation signal. The vertical scale on the graph is logarithmic.

\section{B. System B}

The second optical system was nearly identical to the first except that it employed an opaque Fourier plane mask to block the dc. In this case, the mask consisted of a thin vertical wire located just in front of the MSF. Unfortunately, attenuation filters of this type that are easy to construct, such as binary disks and lines, lead to ringing artifacts. Attenuation filters obtained by photographically recording the illumination system impulse response can be used to avoid this ringing. ${ }^{3}$ 

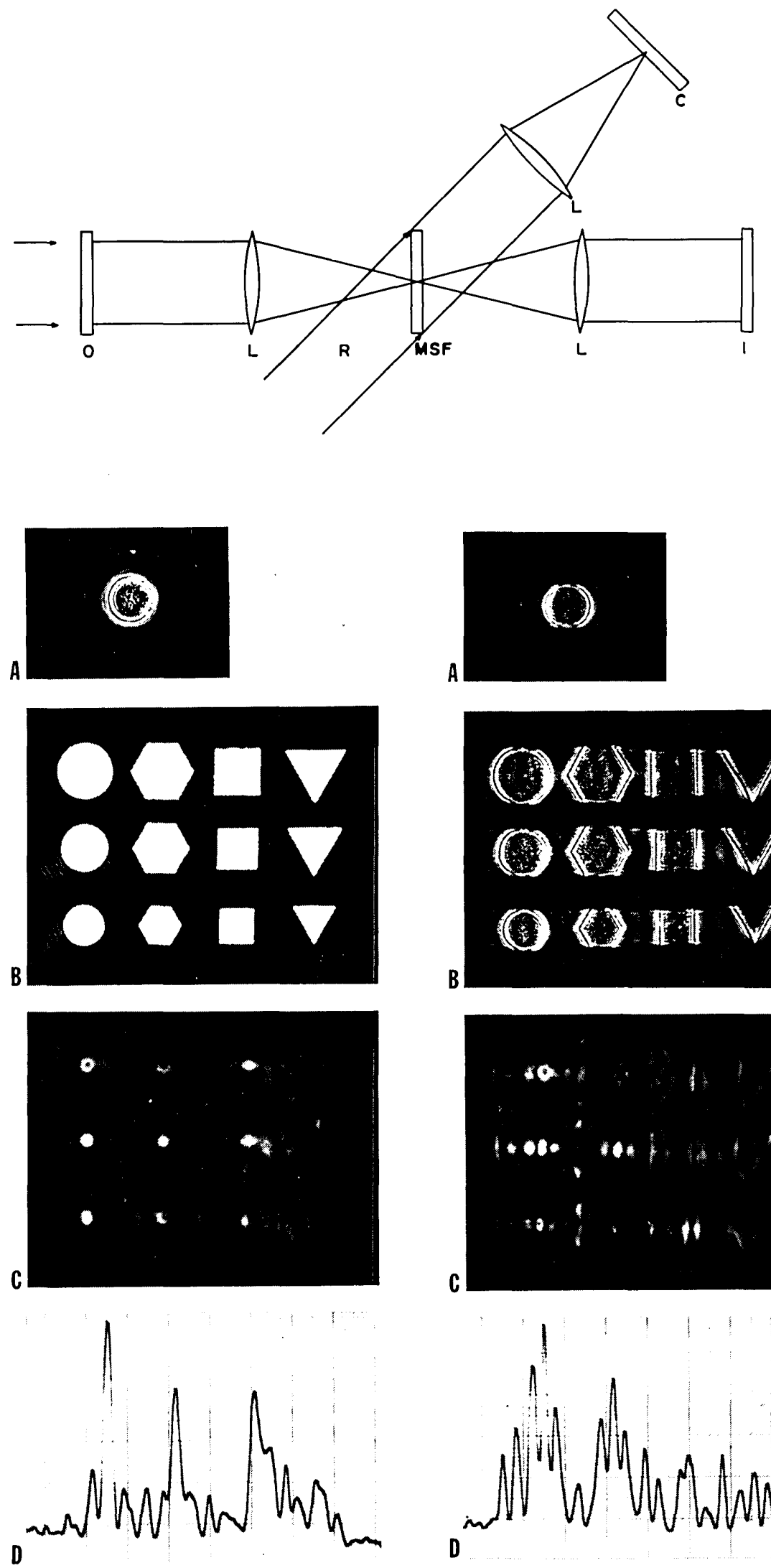

Fig. 2. System A (with overexposed filter): (A) MSF impulse response; (B) input objects; (C) correlation field; (D) scan through middle line of correlation field.

\section{A}
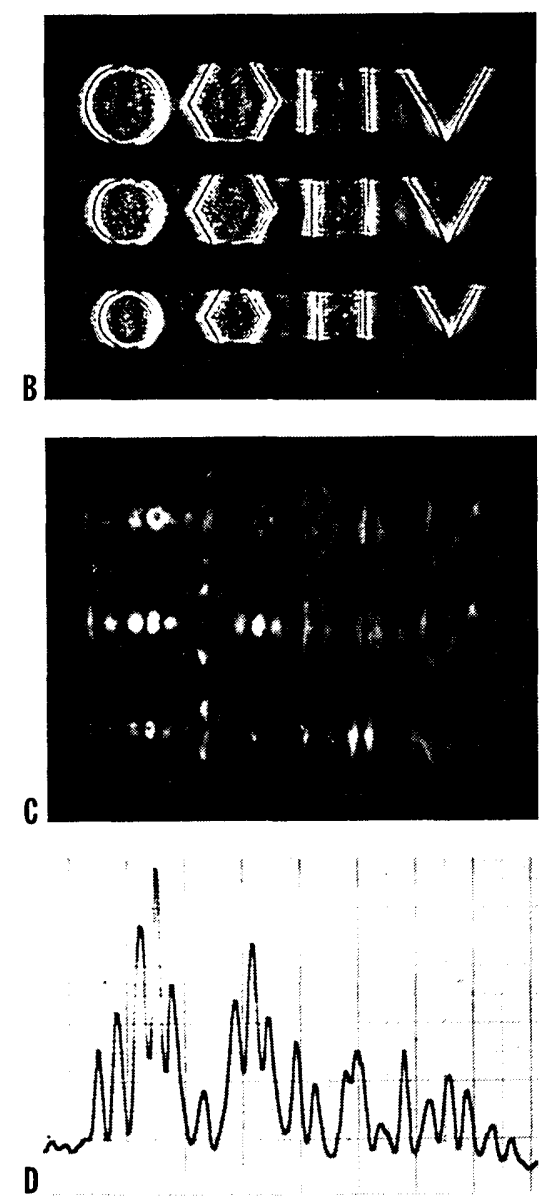

Fig. 3. Same as Fig. 2 except for system B (with wire filter).
Fig. 1. Optical processing system: $O$, input object(s); $R$, reference wave; MSF, matched spatial filter; $I$, image plane; $C$, correlation plane; $L$, Fourier transform lenses.
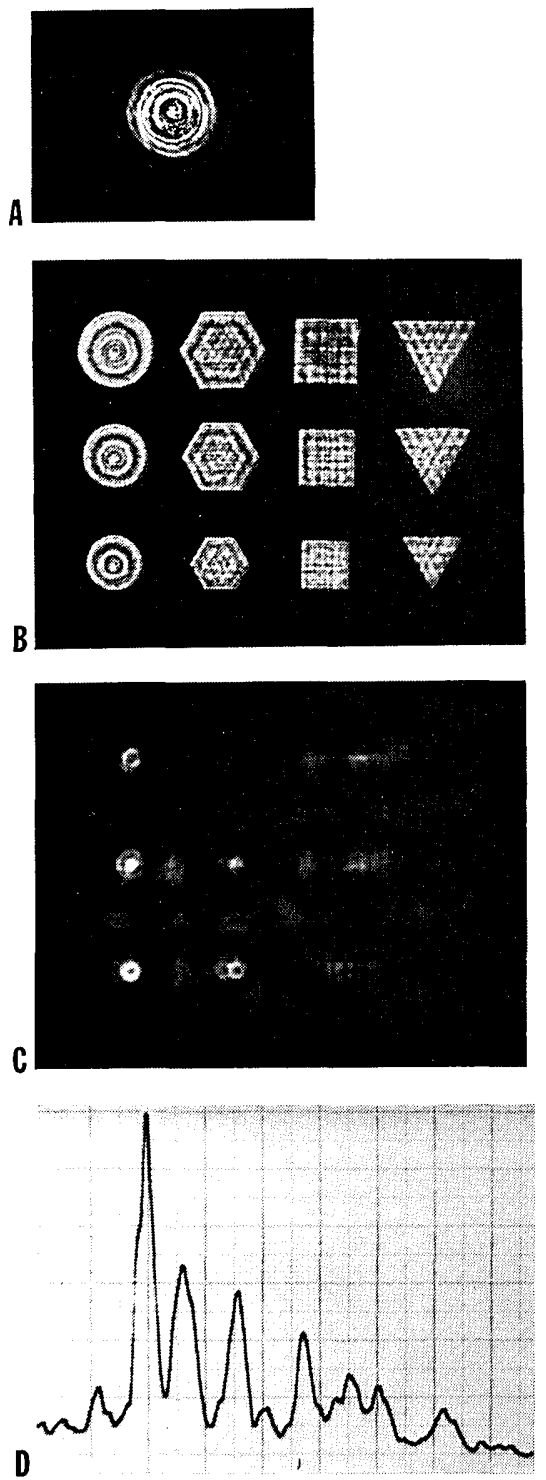

Fig. 4. Same as Fig. 2 except for system $C$ (with Fabry-Perot filter). 
Using the vertical wire preprocessing filter, an MSF of the transparent circle was again constructed such that the filter diffraction efficiency was maximized at the same spatial frequency ( 5 lines $/ \mathrm{mm}$ ) as for the filter in system A. The MSF impulse response is shown in Fig. $3(\mathrm{~A})$. The set of input objects, as viewed through the preprocessing filter, is shown in Fig. 3(B). The correlation plane output for this optical system is shown in Fig. 3(C). An intensity measurement through the second row of output signals [Fig. 3(D)] shows that the hexagon still produces an intense cross-correlation signal.

\section{System C}

This system employs a Fabry-Perot etalon as an object plane preprocessing filter. For mechanical simplicity and to add the possibility of object scaling, the object is actually imaged onto the etalon as shown in Fig. 5.

A Fabry-Perot etalon has a transmittance that is a function of illumination incidence angle. It can thus operate on the angular spectrum of plane waves produced by the object to perform spatial frequency filtering in the object plane. ${ }^{4,5}$

In transmission, a F.P. etalon has intensity transmittance

$$
T(\rho)=\frac{1}{1+\frac{4 F^{2}}{\pi^{2}} \sin ^{2} \delta},
$$

where

$$
F=\pi \sqrt{R} /(1-R)
$$

is the cavity finesse with mirror reflectivity $R$, and

$$
\delta=\frac{2 \pi d}{\lambda} \cos \theta,
$$

where $d$ is the cavity length, $\lambda$ the wavelength, and

$$
\theta=\sin ^{-1}(\lambda \rho)
$$

is the propagation angle of a plane wave diffracted from a normally illuminated object of spatial frequency $\rho$.

For dc suppression, we choose

$$
d / \lambda=(2 N+1) / 4,
$$

with $N=$ integer so that

$$
T(0)=\text { minimum }=\left(1+4 F^{2} / \pi^{2}\right)^{-1} \text {. }
$$

With the above constraint, $T(\rho)$ is plotted for $N=2000$ and various finesse values in Fig. 6. The F.P. preprocessing filter is seen to offer considerable dc suppression for larger finesse values and has its first transmission band centered at $\rho=35$ lines $/ \mathrm{mm}$ with $\lambda=6328 \AA$. The passbands, of course, can be tuned by varying $d$. This has the effect of changing the position of the transmitted bands as well as of changing the dc suppression. The dc transmittance remains low, however, as long as Eq. (5) is approximately satisfied.

We also note that F.P. etalons could be used in reflection with reflectance given by

$$
R(\rho)=\frac{\frac{4 F^{2}}{\pi^{2}} \sin ^{2} \delta}{1+\frac{4 F^{2}}{\pi^{2}} \sin ^{2} \delta} .
$$

Here, the desired constraint $R(0)=$ minimum is satisfied for $d / \lambda=N / 2$. $R(\rho)$ is plotted in Fig. 7 for $N=$ 2000 and various values of finesse. The filter shows very good dc rejection for all finesse values but was experimentally found to be very sensitive to vibrations-a problem that could be eliminated by use of a solid etalon.

A F.P. filter with finesse $F=11$ and $d \approx 0.6 \mathrm{~mm}$ was used in transmission as in Fig. 5. The 1-mm circle used to make the MSF was scaled down by a factor of 4 with the relay system of Fig. 5. The F.P. spacing $d$ was adjusted so that the first transmitted band was located at 20 lines $/ \mathrm{mm}$. With the size reduction, this coincides with the 5 lines $/ \mathrm{mm}$ average spatial frequency used in the two previous systems. An MSF was constructed as for the previous systems. The MSF impulse response is shown in Fig. 4A. The array of input objects, as viewed through the F.P. preprocessing filter, is shown in Fig. 4B. The correlation plane output is shown in Fig. 4C, with the corresponding logarithmic intensity

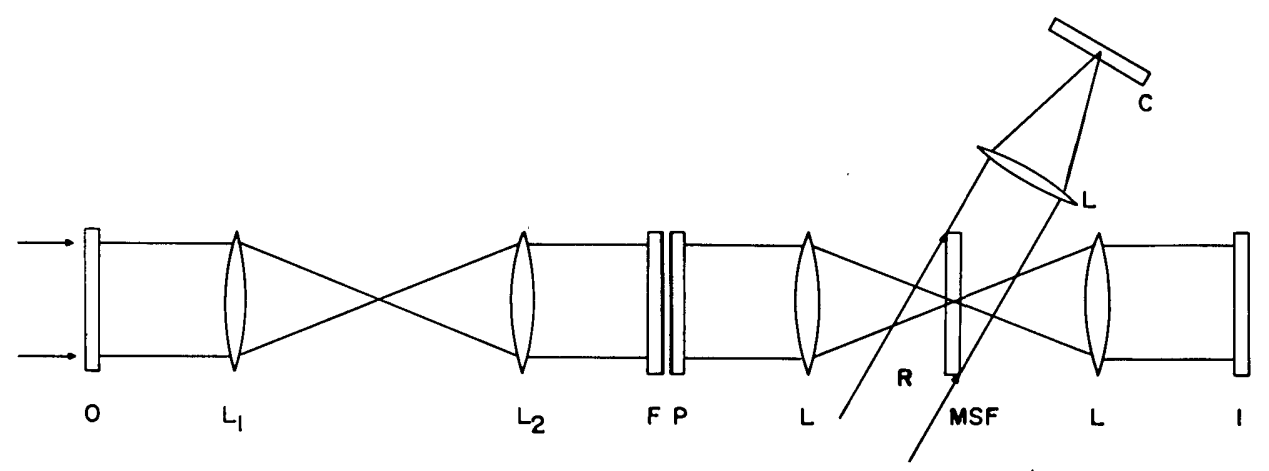

Fig. 5. Optical processing system with F.P. preprocessing filter. Symbols as in Fig. 1 except $L_{1}$ and $L_{2}$ are relay lenses; F.P. = Fabry-Perot etalon. 


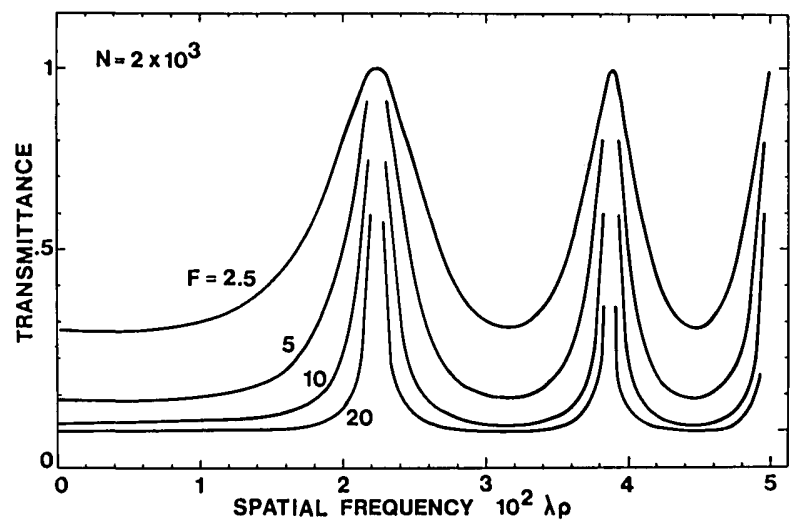

Fig. 6. Transmittance of Fabry-Perot filter: $d=(2 N+1) \lambda / 4$; $N=2000$.

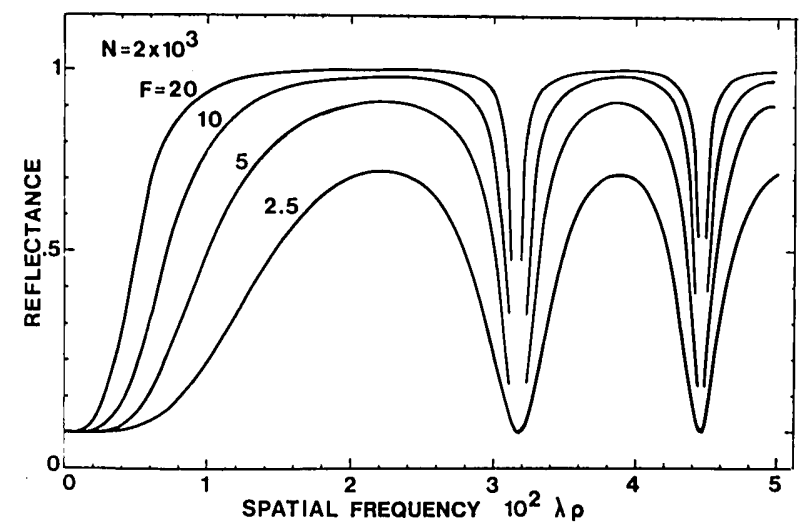

Fig. 7. Reflectance of Fabry-Perot filter: $d=N \lambda / 2 ; N=2000$.

plot in Fig. 7(D). The greater suppression of crosscorrelation signals relative to the autocorrelation signal for this system is evident.

\section{Conclusion}

Three different methods of low spatial frequency suppression were compared in coherent optical systems used for matched spatial filter pattern recognition. A Fabry-Perot etalon placed in the object plane was found to give the best results. The tunable characteristics of Fabry-Perot filters could make them promising for other spatial filtering applications.

\section{References}

1. J. Bulabois, A. Caron, and J. C. Vienot, Opt. Technol. 8, 191 (1969).

2. D. Casasent and A. Furman, Appl. Opt. 15, 1690 (1976).

3. S. K. Case et al., Appl. Phys. 17, 287 (1978).

4. G. Indebetouw, Appl. Opt. 19, 761 (1980).

5. G. Indebetouw, Proc. Soc. Photo-Opt. Instrum. Eng. 232, 224 (1980).

\section{A FLUORESCENCE STANDARD REFERENCE MATERIAL: QUININE SULFATE DIHYDRATE}

\author{
By R. A. Velapoldi and K. D. Mielenz
}

The need, material selection, characterization, certification, and uses of the fluorescence Standard Reference Material, quinine sulfate dihydrate, have been discussed. The emission spectrum for quinine sulfate dihydrate in $0.1 \mathrm{~mol} /$ liter $\mathrm{HClO}_{4}$ has been measured on the NBS reference spectroradiometer. The spectrum has been reported every $5 \mathrm{~nm}$ from 375.0 to $675.0 \mathrm{~nm}$ as the relative technical emission spectrum $E^{\top}(\lambda)$ and the relative molecular emission spectra represented by $E(\lambda), E_{p}(\lambda), E(\tilde{\nu})$, and $E_{p}(\tilde{\nu})$. The technical emission spectrum has been corrected for the instrumental parameters of the spectral responsivity of the detection system, photomultiplier tube nonlinearity, bandpass, monochromator wavelength error, and further corrections for the sample parameters of solvent refractive index and cell window transmittance were applied to obtain the molecular emission spectra. Estimates of the $95 \%$ systematic error limits of the emission spectrum values have been made as a function of wavelength. These values are $\sim 2 \%$ at the peak maximum $(\sim 455 \mathrm{~nm})$ and $\sim 5 \%$ and $\sim 3 \%$ at the blue $(395 \mathrm{~nm})$ and red $(565 \mathrm{~nm})$ one-tenth power points. The experimental replication precision is $\sim 0.2 \%$ over the total spectral range. The purity and the stability of the SRM quinine sulfate dihydrate and the effect of solute concentration, solvent acid, acid concentration, excitation wavelength, oxygen quenching, temperature and polarized exciting radiation on the emission spectrum, and in part, on the absorbance spectrum, photon yield, and fluorescence lifetime also have been discussed.

Copies are available for $\$ 4.25$ from Government Printing Office, Washington D.C. 20402; request SP260-64. 